DOI:

10.1038/nri1930

\title{
Conjugating enzyme IDed
}

Ubiquitin-conjugating enzyme 13 (UBC13) has now been shown to have a key role in the mammalian immune response, in a study published in Nature Immunology. Yamamoto et al. describe how this enzyme is important for B-cell development and for B-cell and macrophage activation.

Previous studies indicated that UBC13 might be important for the polyubiquitylation of TRAF2 (tumour-necrosis factor (TNF)receptor-associated factor 2) and TRAF6, an essential step in the transduction of signals from immune receptors to the key transcription factor nuclear factor- $\kappa \mathrm{B}(\mathrm{NF}-\kappa \mathrm{B})$. To determine the exact mechanism by which UBC13 influences immune signalling, the authors generated mice in which the expression of UBC13 could be conditionally ablated. Using these mice, the authors showed that UBC13 has an important role in the development of marginalzone $\mathrm{B}$ cells and peritoneal $\mathrm{CD}^{+}$ B1 cells, as well as in the activation, cell-cycle progression and viability of splenic B cells, and in the generation of in vivo humoral responses.

To understand the role of UBC13 in response to Toll-like receptor (TLR) activation, UBC13-deficient peritoneal macrophages were stimulated with various TLR ligands. Less TNF, interleukin-6 (IL-6) and IL-12p40 was produced by these cells than by UBC13-sufficient cells. Surprisingly, activation of NF- $\kappa \mathrm{B}$ was not markedly affected. However, in the absence of UBC13, activation of members of the mitogen-activated protein kinase (MAPK) family was severely impaired in response to various stimuli, including TLR ligands and IL- $1 \beta$, but not TNF.
To understand at which point in the signal-transduction pathway UBC13 was having an effect, the authors then examined TLR- or IL-1 $\beta$-induced polyubiquitylation of some of the signalling molecules involved. Polyubiquitylation of TRAF6 was similar in both UBC13-deficient mouse embryonic fibroblasts (MEFs) and UBC13-sufficient MEFs. IL-1 $\beta$-induced activation of TAK1 (transforming-growth-factor- $\beta$ activated kinase 1 ), which is activated by TRAF6 and is known to activate MAPKs, was slightly affected by the absence of UBC13 in MEFs. However, the authors found that UBC13dependent polyubiquitylation of IKK $\gamma$ (inhibitor-of-NF- $\kappa B$ kinase $\gamma$ ) might be involved in the activation of members of the MAPK family. Further studies are now needed to elucidate more fully the involvement of IKK $\gamma$ in the activation of MAPKs and NF- $\kappa \mathrm{B}$.

So this study shows that $\mathrm{UBC} 13$ is involved in the activation of MAPKs, in the generation and activation of $\mathrm{B}$ cells, and in the activation of macrophages, indicating that this enzyme has an important role in immune responses.

\section{Olive Leavy}

ORIGINAL RESEARCH PAPER Yamamoto, M. et al. Key function for the Ubc13 E2 ubiquitinconjugating enzyme in immune receptor signaling. Nature Immunol. 23 July 2006 (doi:10.1038/ni1367) 